\title{
Two-dimensional satellite image compression using compressive sensing
}

\author{
Gunasheela Keragodu Shivanna, Haranahalli Shreenivasamurthy Prasantha \\ Department of Electronics and Communication Engineering, Nitte Meenakshi Institute of Technology, Bengaluru, India
}

\begin{abstract}
Compressive sensing is receiving a lot of attention from the image processing research community as a promising technique for image recovery from very few samples. The modality of compressive sensing technique is very useful in the applications where it is not feasible to acquire many samples. It is also prominently useful in satellite imaging applications since it drastically reduces the number of input samples thereby reducing the storage and communication bandwidth required to store and transmit the data into the ground station. In this paper, an interior point-based method is used to recover the entire satellite image from compressive sensing samples. The compression results obtained are compared with the compression results from conventional satellite image compression algorithms. The results demonstrate the increase in reconstruction accuracy as well as higher compression rate in case of compressive sensing-based compression technique.
\end{abstract}

This is an open access article under the $\underline{C C B Y-S A}$ license.

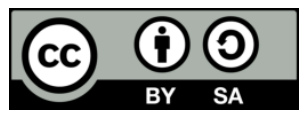

\section{Corresponding Author:}

Gunasheela Keragodu Shivanna

Department of Electronics and Communication Engineering, Nitte Meenakshi Institute of Technology

Bengaluru, Karnataka, India

Email: gunasheela.ks.2012@gmail.com

\section{INTRODUCTION}

Image processing applications plays a vital role in the present digital world. In that satellite imaging has many useful applications in agriculture, remote sensing, urban planning and mineral exploration. Despite many useful applications, it is critical to transmit the satellite image data into the ground station in the limited transmission bandwidth. The satellite will be in contact with the ground station only at certain intervals. The data need to be stored on-board until the next communication window of the satellite. For all the reasons, it is very important to compress the satellite image data to make the communication and storage feasible. Image compression algorithms are broadly classified into lossless and lossy image compression algorithms. The satellite image is not only used for viewing purposes but also used to make important exploratory analysis. Therefore, lossless image compression [1] is the modality used in most of the applications. But in certain cases, to improve the image acquisition rates and to improve the compression rate lossy satellite image compression algorithms are made use in missions like SPOT 4 [2].

Nyquist sampling theorem [3] is the well-known method for signal acquisition and faithful recovery of signal from the measurements. Advances in compressive sensing-based methodologies gives satisfactory proof on retrieving the signal from a smaller number of samples when the signal is compressible in nature. The modality of compressive sensing is very useful when it is not practical to acquire many samples and when it is very expensive to collect large number of samples. In both cases compressive sensing can contribute by making the image acquisition hardware simpler, reducing the image acquisition time, and thereby achieving image compression. 
Conventional image compression algorithms rely on the Nyquist sampling theorem where large numbers of samples are collected only to be discarded at the stage of compression. Conventional image compression algorithms in the literature are generally based on transform coding techniques. Where, the images are transformed using discrete cosine transform (DCT) or discrete wavelet transform (DWT). The coefficients are then encoded using encoding techniques like run-length encoding, Golomb coding [4], [5], Arithmetic coding [6], and rice coding [7]. Many conventional image compression algorithms have been proposed in the literature for the compression of satellite images. The consultative committee for space data systems (CCSDS) document [8] describes the lossless compression methods for satellite images. The image compression algorithm based on DWT called set partition in hierarchical trees (SPIHT), where the significant DWT coefficients are encoded first for the fast and efficient coding of images is presented in [9]. Aranki et al. [10], the field programmable gate arrays (FPGA) implementation of the lifting scheme of DWT is proposed. The research work in [10] and [11] discuss the concept of finding the DWT coefficients of an image segment, in a pipeline fashion, instead of waiting for the entire image from the push-broom scanning, since the values of DWT coefficients depends on the pixels in the neighbourhood. JPEG-LS algorithm for the lossless compression of images is presented in [12]. A low complexity context based lossless image compression algorithm is presented in [13]. The JPEG-2000 algorithm, which is a DWT version of DCT based JPEG to achieve different degrees of image quality based on different applications is proposed by the joint photographic experts' group in [14]-[16]. Compressive sensing [17]-[19] is based on the principle that if the signal is compressible in nature, it is possible to reconstruct the signal from very few measurements. The compressive samples are generated using the random sampling matrix which captures the signal in very few measurements.

Figure 1 shows the general block diagram of the conventional image compression algorithms like JPEG and CCSDS standard. As it is evident from the block diagram, the satellite image goes through many processing steps at the encoder stage. First the image is transformed using one of the transform coding techniques like DCT or DWT. The insignificant coefficients are discarded after the transform coding. The remaining coefficients are subjected to quantization in the next stage. The quantized coefficients are further encoded using entropy coding techniques like Huffman coding, and arithmetic coding. The encoder is very simple in case of CS based compression algorithms as shown in Figure 2. The only processing step at the encoder is generation of compressive sensing samples from the random compressive sensing matrix. The decrease in computational steps in the encoder is of great significance in case of remote sensing application because memory and power are the two important critical resources in case of any remote sensing application.

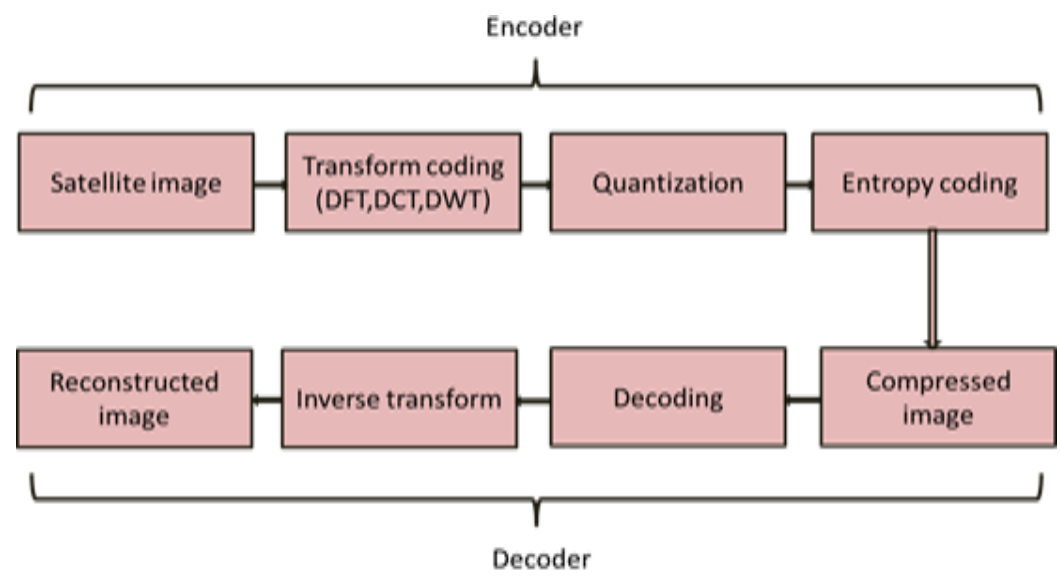

Figure 1. General block diagram of the conventional satellite image compression algorithm

The most significant aspect of using CS for the compression in remote sensing applications can be attributed to the property of making the encoder very simple by removing all the processing steps in the encoder stage of the conventional compression algorithm. The compressive sensing samples are communicated to the ground station in case of satellite imaging applications. The disadvantage of CS based algorithms for compression is that the reconstruction process from compressive sensing samples is quite computationally expensive. This aspect can be overlooked as the reconstruction process happens in the ground station where resources are available in abundance. 
The research work makes use of compressive sensing techniques to compress the satellite images. Compressive sensing samples are generated using a CS matrix containing random Fourier transform coefficients. The satellite image is compressed at various sampling rates to analyse the performance of the algorithm at different levels of compression. The satellite image is reconstructed from the compressive sensing samples by a class of interior point methods called log barrier method. The iterative technique minimizes the objective function with total variation regularization to solve the problem of image reconstruction from compressive sensing samples. The rest of the article is organized as follows; Section 2 describes the compressive sensing technique proposed to reconstruct the satellite image from compressive sensing samples. Section 3 presents the experimental results using different datasets. The performance of the algorithm is analysed using quantitative metrics like peak signal to noise ratio (PSNR) and structural similarity index measure (SSIM). Section 4 concludes the research work presented in the article.

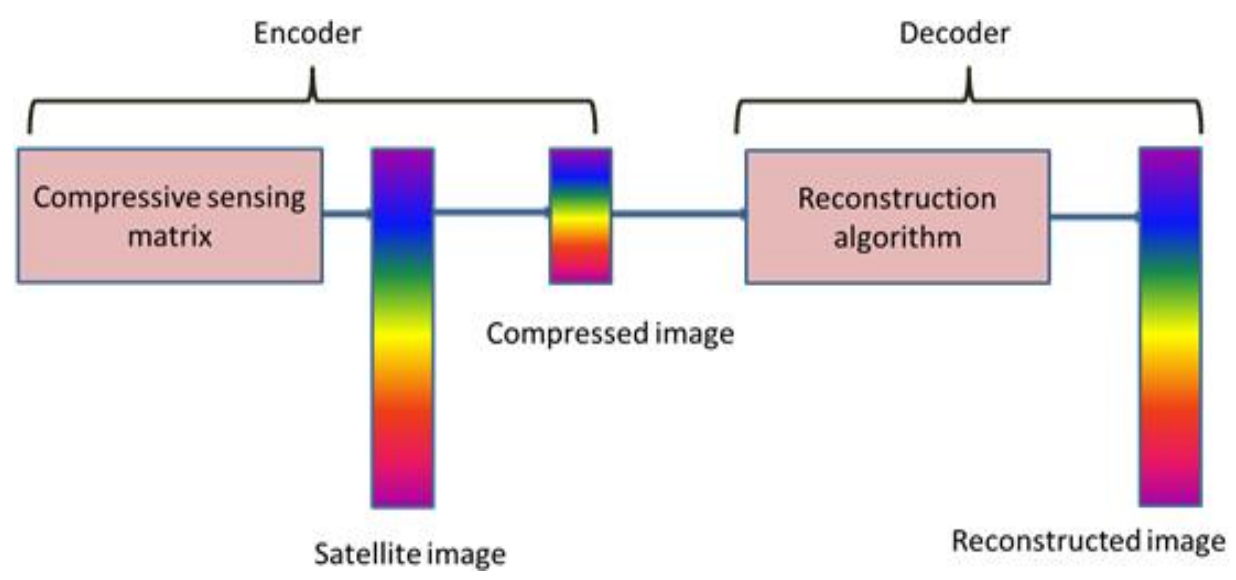

Figure 2. General block diagram of compressive sensing-based algorithm

\section{CS-CR ALGORITHM}

Consider an image $I$, which has dimensions $n \times n$ The total number of pixels in $I$ is denoted by $N$. The image $I$ is vectorized as $x:=\operatorname{Vec}(I)$, where the length of the vector $x$ is $N$. The results in papers [20]-[22] demonstrates the possibility of recovering signals from fewer number of measurements i.e., $z=C x \in R^{P}, P \ll N$ with considerable accuracy. The basis pursuit problem to solve for optimal value of $x$ from fewer compressive sensing samples $z$ is given by (1):

$$
\min _{x}\|x\|_{1} \text { such that } C x=z
$$

The optimization problem solves for $x$ with minimum $l_{1}$ norm, which explains the compressive sensing measurements $z$. Another strategy to solve for $x$ is given by (2):

$$
\min _{x} T V(x) \text { such that } C x=z
$$

where, $T V(x)$ represents the total variation [23] of $x . T V(x)$ is defined as (3),

$$
T V(x)=\sum_{i, j} \sqrt{\left(D_{i, j}^{h} I\right)^{2}+\left(D_{i, j}^{v} I\right)^{2}}
$$

where,

$$
\begin{aligned}
& D_{i, j}^{h} I= \begin{cases}I_{i+1}-I_{i, j} & i<n \\
0 & i=n\end{cases} \\
& D_{i, j}^{v} I= \begin{cases}I_{i, j+1}-I_{i, j} & j<n \\
0 & j=n\end{cases}
\end{aligned}
$$

The problem in (2) can be generalized as (6), 
$\min _{x}<k_{o}, x>$ such that $C x=z, F_{i}(x) \leq 0 \quad i=1 \ldots . m$

where, $F_{i}$ represents the $m$ linearity constraints. The optimization problem in (6) can be converted into a series of sub problems with linearity constraints using the Log-barrier method [24] as (7):

$$
\min _{x}<k_{o}, x>+\frac{1}{\tau^{L}} \sum_{i}-\log \left(-F_{i}(x)\right) \text { such that } C x=z
$$

Log-barrier method augments the inequality constraints into the objective by log penalty function. At each iteration $L$, the value of $\tau$ is increased. When $\tau$ is sufficiently large, the solution $x^{L}$ approaches the optimal value of the original problem in (6). The second order approximation of (7) at $\Delta x$ is given by (8):

$$
F_{0}(x+\Delta x) \approx x+<G r_{x}, \Delta x>+\frac{1}{2}<H_{x} \Delta x, \Delta x>
$$

where, $G r_{x}$ represents the gradient, which is given by (9):

$$
G r_{x}=k_{0}+\frac{1}{\tau} \sum_{i} \frac{1}{-F_{i}(x)} \nabla F_{i}(x)
$$

$H_{x}$ Represents the Hessian matrix, which is given by (10):

$$
H_{x}=\frac{1}{\tau} \sum_{i} \frac{1}{F_{i}(x)^{2}} \nabla f_{i}(x)\left(\nabla F_{i}(x)\right)^{T}+\frac{1}{\tau} \sum_{i} \frac{1}{-F_{i}(x)} \nabla^{2} F_{i}(x)
$$

If $x$ is in feasible set, $\Delta x$ which represents the minima of second order approximation in (8) is given by the following set of linear:

$$
\tau\left(\begin{array}{cc}
H_{x} & C^{T} \\
C & 0
\end{array}\right)\left(\begin{array}{c}
\Delta x \\
\rho
\end{array}\right)=-\tau G r_{x}
$$

$\rho$ is the Lagrange multiplier for the equality constraints.

The flowchart of the CS-CR algorithm based on Log-barrier method for the reconstruction of image from compressive sensing measurements is given in Figure 3. The algorithm is initialized with $x^{o}$, tol, $L, \tau, \alpha$ in step 1. In step 2 (7) is solved using Newton method using $x^{L-1}$ as initial point. In step 3 , if $\frac{m}{\tau_{L}}<$ tol, algorithm is terminated, else the algorithm goes back to step 2 .

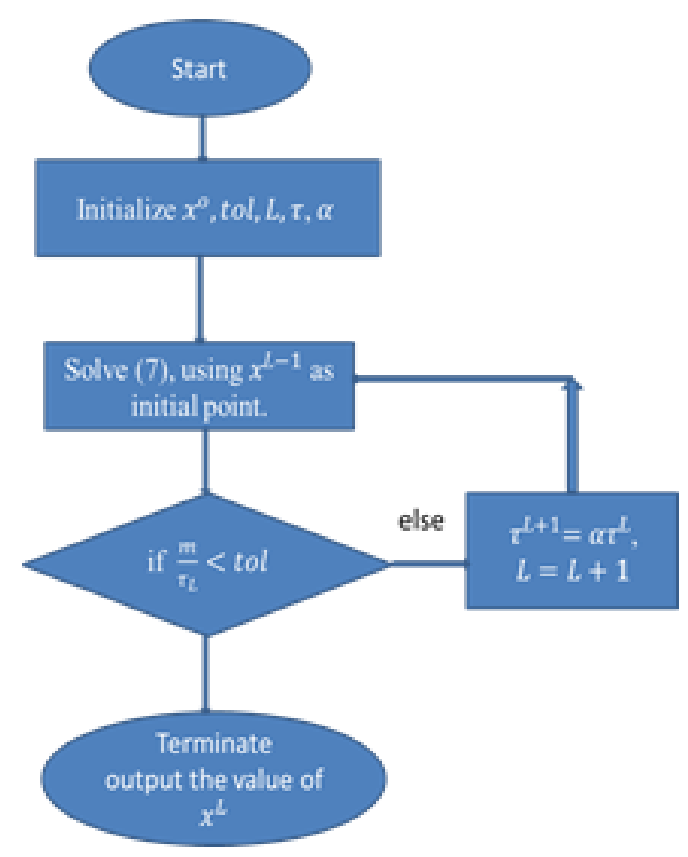

Figure 3. Flowchart of the CS-CR algorithm 


\section{RESULTS AND DISCUSSION}

Experiments are conducted using MATLB R2016b in windows 10 laptop, 64-bit operating system with Intel(R) i7 processor, 16 GB RAM. For the experimentation two datasets [25] have been considered. A band from coastal_b1 data of Landsat NASA is considered as the first data. The image is $1024 \times 1024$ in size. Another data considered for experimentation is spot_la_b3 from SPOT 3 CNES. The image is $500 \times 500$ in size. Three algorithms [26] are considered to compare and validate the performance of the CS-based reconstruction algorithm. The algorithm proposed in CCSDS document, uses float DWT to achieve lossy compression of satellite images, where the parameters are tuned to achieve different rate distortion performance and full frame compression. The JPEG-2000 algorithm based on float DWT in both scan mode and frame mode is considered for the comparison where rate allocation optimization is done using the Lagrange multipliers. Another algorithm considered for comparison is the set partitioning in hierarchical trees (SPIHT), which is based on float DWT to down sample the image into different decomposition levels. The methods are compared in terms of quantitative metrics like PSNR and SSIM. PSNR provides the information about the average numerical similarity between the original and reconstructed image. SSIM provides the measure of average spatial structural similarity between original and reconstructed image. The measures PSNR and SSIM are defined as follows:

- Peak signal to noise ratio (PSNR):

$$
\operatorname{PSNR}(X, \hat{X})=20 \log _{10} \frac{\max (X)}{\sqrt{\operatorname{MSE}(X, \hat{X})}}
$$

$X$ and $\hat{X}$ are the original image and the reconstructed image, respectively.

- Mean square error (MSE):

$$
\operatorname{MSE}(X, \hat{X})=\frac{1}{N}\|X-\hat{X}\|_{2}^{2}
$$

- Structural similarity index measure (SSIM):

$$
\operatorname{SSIM}(X, \hat{X})=\frac{\left(2 \mu_{X} \mu_{\widehat{X}}+C_{1}\right)\left(2 \sigma_{x \widehat{x}}+K_{2}\right)}{\left(\mu_{X}^{2}+\mu_{\widehat{X}}^{2}+C_{1}\right)\left(\sigma_{X}^{2}+\sigma_{\widehat{X}}^{2}+K_{2}\right)}
$$

$\mu_{X}$ and $\mu_{X}$ refers to the mean values of original image and reconstructed image, respectively. $\sigma_{X}$ and $\sigma_{X}$ refers to the standard deviations of the original and reconstructed image, respectively. $K_{1}$ and $K_{2}$ are the constants related to the pixel values dynamic range. Higher PSNR and SSIM is the indication for increase in reconstruction accuracy. PSNR and SSIM are evaluated at different sampling rates for the CS-based algorithm. Sampling rate is the proportion of samples extracted from the original image with respect to total number of samples in the original image.

Figure 4 shows the reconstruction results using CS-based algorithm for coastal_b1 Landsat data at various sampling rates ranging from 0.1 to 0.5 . Similarly Figure 5 shows the reconstruction results using CSbased algorithm for spot_la_b3 data at various sampling rates ranging from 0.1 to 0.5 . Figure 6(a) provides the comparison of PSNR values for two datasets spot_la_b3 and coastal_b1 using conventional compression techniques like JPEG-2000, SPIHT and CCSDS and the proposed CS- based technique. It is evident from the graph that, PSNR achieved using CS-based algorithm is much higher in case of both the datasets.

The average PSNR value using JPEG-2000 algorithm for the spot_la_b3 dataset is 42.21 . The average PSNR value for the same dataset using CCSDS based algorithm is 41.84. Similarly, the PSNR value using SPIHT algorithm is given by 41.68 . The PSNR value achieved using the proposed compressive sensing-based algorithm is 74.82 for the spot_la_b3 dataset, which is $77 \%, 78 \%, 79 \%$ better compared to JPEG-2000, CCSDS, and SPIHT algorithms, respectively. The average PSNR value using JPEG-2000 algorithm for the coastal_b1 dataset is 51.39. The average PSNR value for the same dataset using CCSDS based algorithm is 51.05. Similarly, the PSNR value using SPIHT algorithm is given by 50.64. The PSNR value achieved using the proposed compressive sensing-based algorithm is 93.67 for the coastal_b1 dataset, which is $82.27 \%, 83.48 \%$, 84.97\% better compared to JPEG-2000, CCSDS, and SPIHT algorithms, respectively. Figure 6(b) provides the PSNR values using CS based compression technique at different sampling rates. It is evident that there is a steady increase in the PSNR values with increase in the sampling rate for both datasets. The PSNR at 0.1 sampling rate for spot_la_b3 data is 72.68. The PSNR at 0.1 sampling rate for coastal_b1 data is 90.97 . Figure 6(c) provides the structural similarity index measure for the two datasets considered at various sampling rates using CS-based compression technique. Average SSIM value in case of coastal_b1 data is 0.9984 . Average SSIM value in case of spot_la_b3 data is 0.9961. Figure 6(d) provides the run time of the CS-based compression technique at various sampling rates. The average run time 
for the spot_la_b3 data is 341.97 s. The average run time for the coastal_b1 data is $1027 \mathrm{~s}$. Decrease in run time with increase in the sampling rate indicates that the algorithm converges faster when a greater number of samples are available as input. Figure 6(e) and Figure 6(f) provides the compression size in bytes of the original and reconstructed image at various sampling rates using CS-based compression technique for the datasets coastal_b1 and spot_la_b3, respectively.
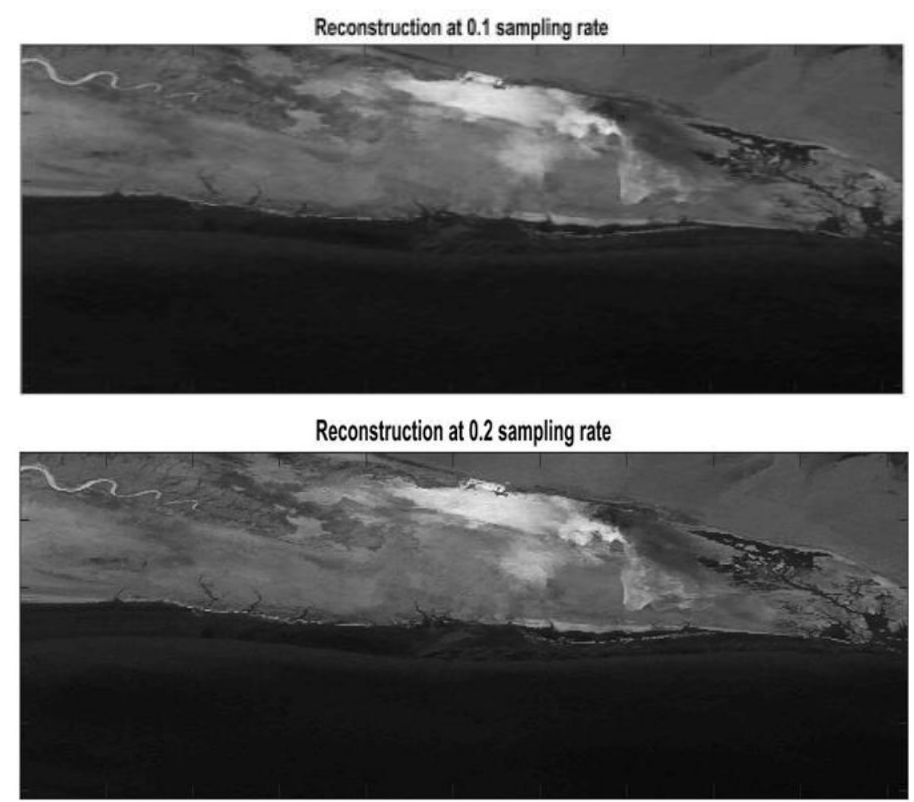

Reconstruction at 0.3 sampling rate

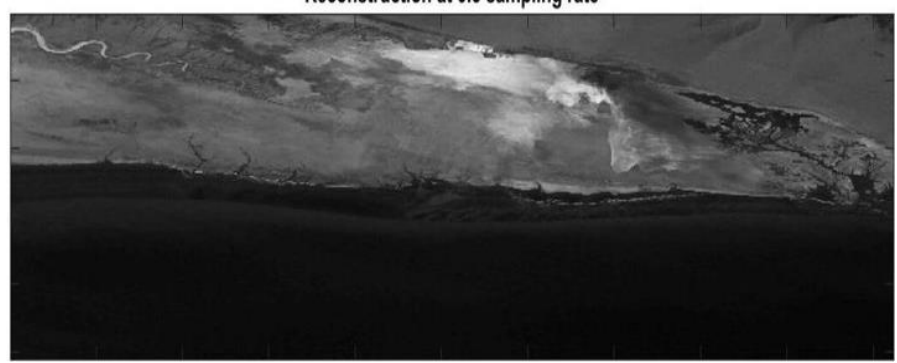

Reconstruction at 0.4 sampling rate

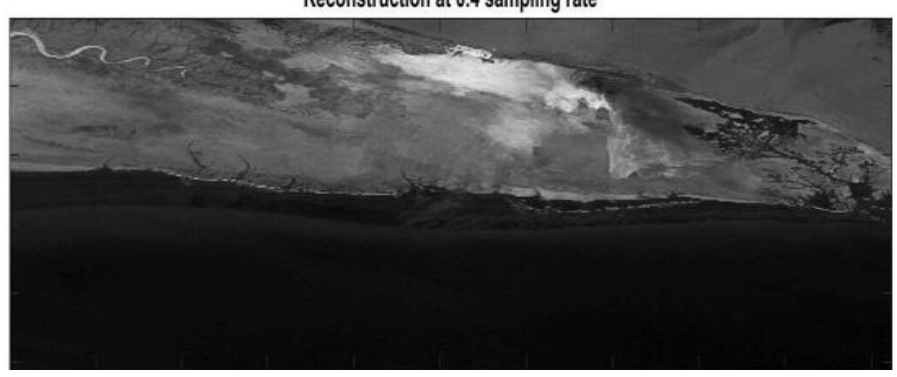

Reconstruction at 0.5 sampling rate

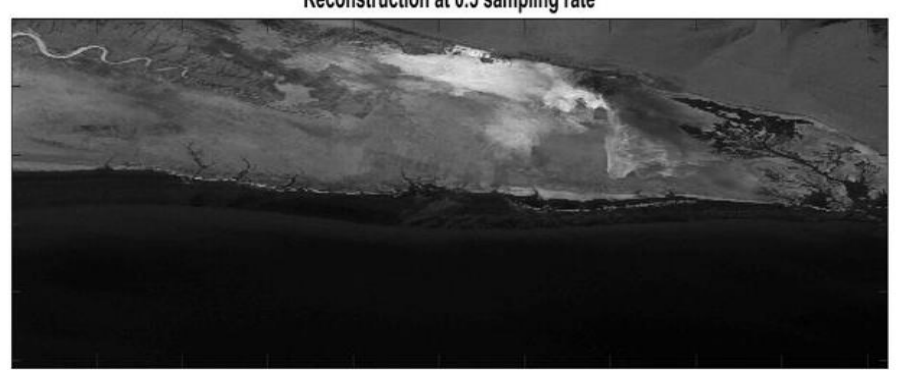

Figure 4. Reconstructed image at $0.1,0.2,0.3,0.4,0.5$ sampling rates respectively, coastal_b1 Landsat data 


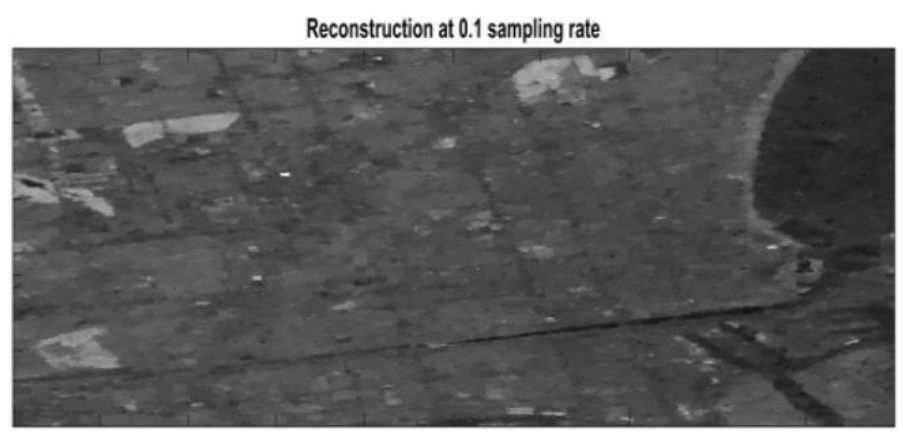

Reconstruction at 0.2 sampling rate

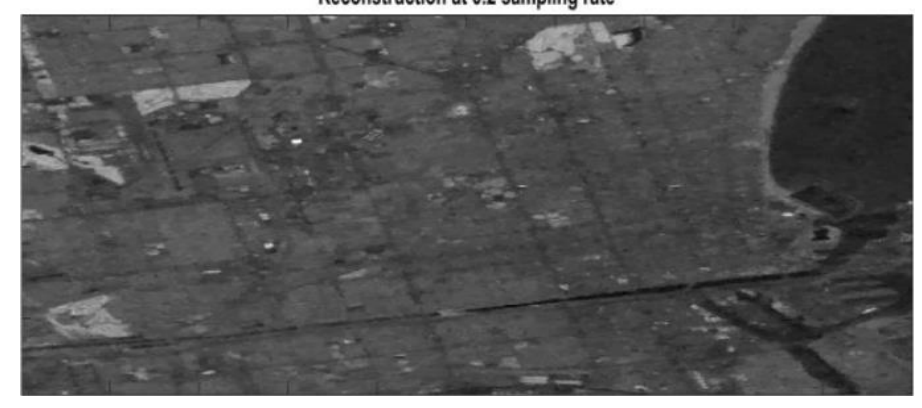

Reconstruction at 0.3 sampling rate

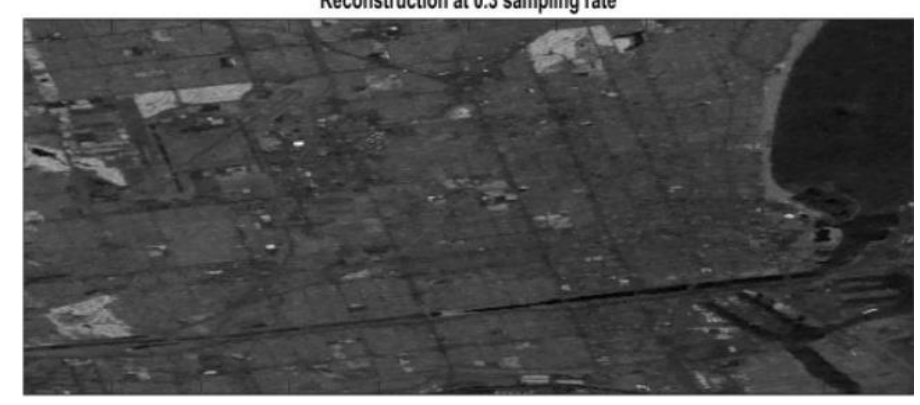

Reconstruction at 0.4 sampling rate
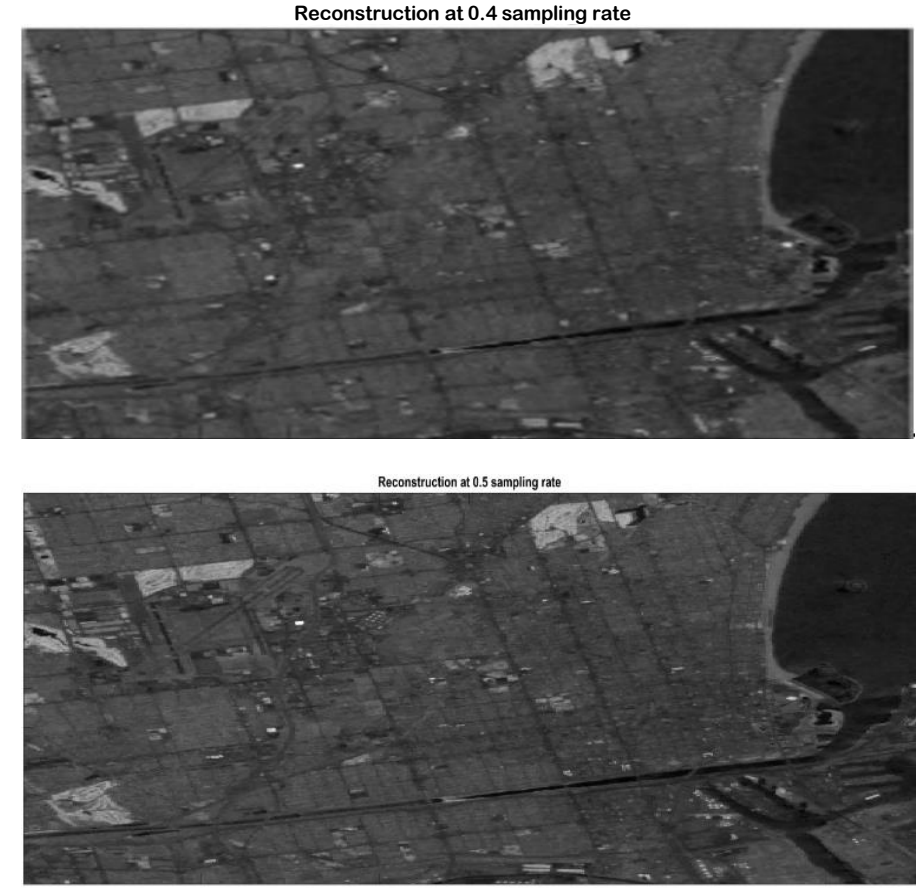

Figure 5. Reconstructed image at 0.1, 0.2, 0.3, 0.4, 0.5 sampling rates respectively, spot_la_b3 CNES data [25] (All rights reserved (C) CNES (2007)) 


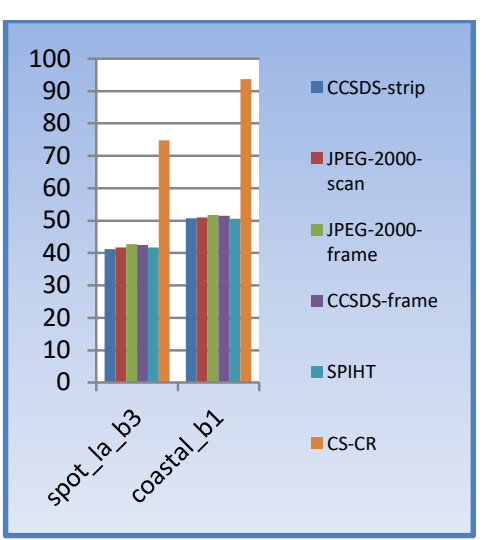

(a)

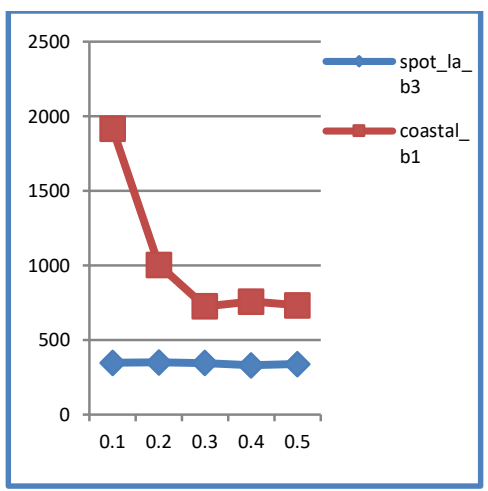

(d)

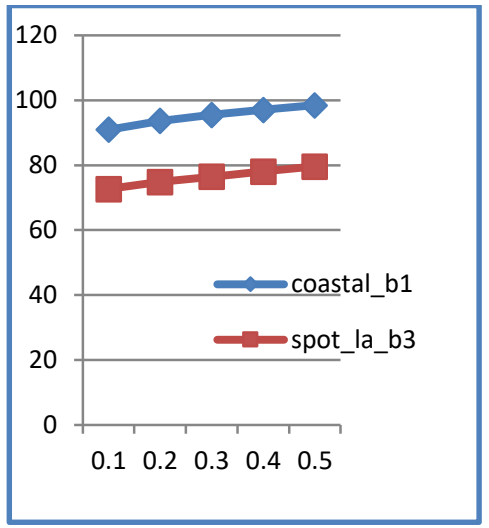

(b)

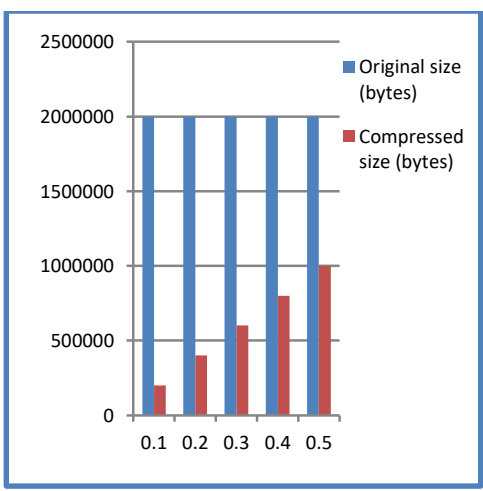

(e)

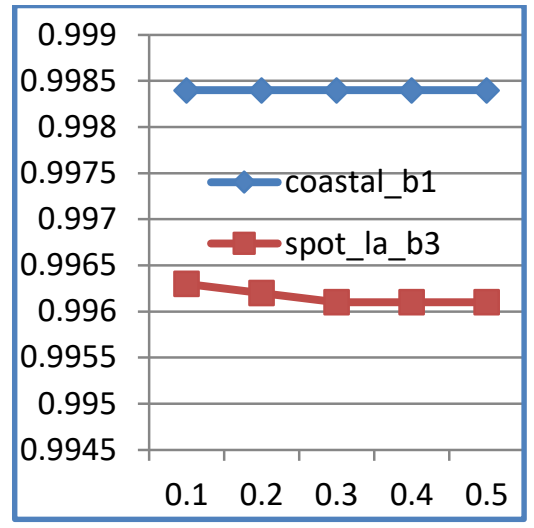

(c)

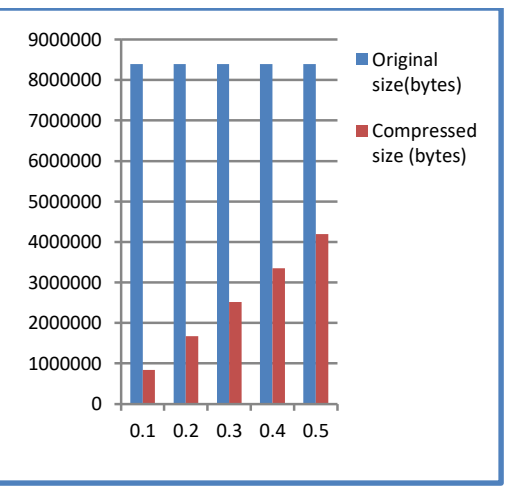

(f)

Figure 6. These figures are; (a) PSNR using different compression techniques, (b) PSNR at various sampling rates using CS based compression technique, (c) SSIM at various sampling rates using CS based compression

technique, (d) run time in seconds at various sampling rates using CS based compression technique,

(e) original and compressed size of coastal_b1 image at different sampling rates, (f) original and compressed size of spot_la_b3 CNES image at different sampling rates

\section{CONCLUSION}

The main goal of this research work is to demonstrate the usefulness of the compressive sensingbased compression techniques in satellite image compression domain. The experimental results and analysis demonstrate the supremacy of the compressive sensing-based technique over the conventional image compression techniques both in terms of the reconstruction accuracy and compression rate. The compressive sensing techniques will be extremely useful in compression of huge hyperspectral and multispectral satellite image datasets as they greatly alleviate the storage and transmission bandwidth bottlenecks in remote sensing applications.

\section{REFERENCES}

[1] Gunasheela K. S. and H. S. Prasantha, "Satellite image compression-detailed survey of the algorithms," Proceedings of ICCR in LNNS Springer, vol. 14, 2017, pp. 187-198, doi: 10.1109/ICPADS.2012.35.

[2] C. Lambert-Nebout and G. Moury, "A survey of on-board image compression for CNES space missions," in: Proceedings of the 1999 IEEE International Geoscience and Remote Sensing Symposium, 1999, pp. 2032-2034, doi: 10.1109/IGARSS.1999.775023.

[3] R. J. Marks (II), "Introduction to Shannon sampling and interpolation theory," Springer-Verlag, 1991.

[4] S. Golomb, "Run-length encodings," IEEE Transactions on Information Theory, vol. 12, no. 3, pp. 399- 401, Jul. 1966.

[5] A. Kiely, "Selecting the golomb parameter in rice coding," The Interplanetary Network Progress Report, vol. 42, no. 159, Nov. 2004.

[6] D. Le Gall and A. Tabatabai, "Sub-band coding of digital images using symmetric short Kernel filters and arithmetic coding techniques," In Proceedings of International Conference on Acoustics, Speech, and Signal Processing, 1988 (New York, NY, USA), vol. 2, pp. 761-764, Apr. 1988, doi: 10.1109/ICASSP.1988.196696.

[7] R. F. Rice, "Some Practical Universal Noiseless Coding Techniques," NTRS-NASA technical reports server Pasadena, California: JPL, United State, Rep. JPL-PUB-79-22; NASACR-158515, 1979.

[8] Lossless Data Compression, Recommendation for Space Data System Standards, CCSDS 121.0-B-3, Blue Book, Issue 1, Washington, D.C.: CCSDS, May 1997. 
[9] A. Said and W. A. Pearlman, "A New, Fast, and Efficient Image Codec Based on Set Partitioning in Hierarchical Trees," IEEE Transactions on Circuits and Systems for Video Technology, vol. 6, no. 3, pp. 243-250, Jun 1996, doi: 10.1109/76.499834.

[10] N. Aranki, W. Jiang, and A. Ortega, "FPGA-Based Parallel Implementation for the Lifting Discrete Wavelet Transform," In Parallel and Distributed Methods for Image Processing IV, vol. 4118, pp. 96-107, 2000, doi: 10.1117/12.403593.

[11] C. Chrysafis and A. Ortega, "Line-Based, Reduced Memory, Wavelet Image Compression," IEEE Transactions on Image Processing, vol. 9, no. 3, pp. 378-389, Mar. 2000, doi: 10.1109/83.826776.

[12] M. J. Weinberger, G. Seroussi, and G. Sapiro, "The LOCO-I Lossless Image Compression Algorithm: Principles and Standardization into JPEG-LS," IEEE Transactions on Image Processing, vol. 9, no. 8, pp. 1309-1324, Aug. 2000, doi: 10.1109/83.855427.

[13] M. J. Weinberger, G. Seroussi, and G. Sapiro, "LOCO-I: A Low Complexity, Context Based, Lossless Image Compression Algorithm," In Proceedings of Data Compression Conference, 1996 (Snowbird, UT, USA), 1996, pp. 140-149, doi: 10.1109/DCC.1996.488319.

[14] D. Taubman and M. Marcellin, "JPEG2000: Image Compression Fundamentals, Standards and Practice," Kluwer International Series in Engineering and Computer Science, Norwell, Massachusetts: Kluwer, November 2001.

[15] M. D. Adams and F. Kossentini, "JasPer: A Software-Based JPEG-2000 Codec Implementation," In Proceedings of 2000 International Conference on Image Processing (Vancouver, BC, Canada), vol. 2, 2000, pp. 53-56, doi: 10.1109/ICIP.2000.899223.

[16] Information Technology-JPEG 2000 Image Coding System: Core Coding System, International Standard, ISO/IEC 15444-1:2004. 2nd ed. Geneva: ISO, 2004.

[17] M. Lustig, D. Donoho and J. Pauly, "Sparse MRI: The application of compressive sensing for rapid MR imaging," Magnetic Resonance in Medicine, vol. 58 no. 6 pp. 1182-1195, 2007, doi: 10.1002/mrm.21391.

[18] D. L. Donoho, "Compressive sensing," IEEE Transactions on Information Theory, vol. 52, no. 4, pp. 1289-1306, 2006.

[19] Gunasheela K. S., H. S. Prasantha, "Compressive sensing for image compression: survey of algorithms," Proceedings of Emerging Research in Computing, Information, Communication and Applications, ERCICA, Springer publication, Bengaluru, 2018.

[20] E. Cand es and J. Romberg, "Quantitative robust uncertainty principles and optimally sparse decompositions," To appear in Foundations of Comput. Math., 2005.

[21] E. Cand'es, J. Romberg, and T. Tao, "Robust uncertainty principles: Exact signal reconstruction from highly incomplete frequency information," Submitted to IEEE Trans. Inform. Theory, Jun. 2004, doi: 10.1109/TIT.2005.862083.

[22] E. Cand es, J. Romberg, and T. Tao, "Stable signal recovery from incomplete and inaccurate measurements," Communications on Pure and Applied Mathematics, 2005, doi: 10.1002/cpa.20124.

[23] T. Chan, G. Golub, and P. Mulet, "A nonlinear primal-dual method for total variation-based image restoration," SIAM J. Sci. Comput., vol. 20, no. 6, 1999, doi: 10.1137/S1064827596299767.

[24] S. Boyd and L. Vandenberghe, Convex Optimization, Cambridge University Press, 2004.

[25] Y. P. Shu, Apr 27, 2007, "Test image datasets," CWE CCSDS. [Online]. Available: https://cwe.ccsds.org/sls/docs/Forms/AllItems.aspx?RootFolder=\%2fsls $\% 2$ fdocs $\% 2 \mathrm{fSLS} \% 2 \mathrm{dDC} \% 2 \mathrm{fBB} 122 \mathrm{TestImage} \&$ FolderC $\mathrm{TID}=0 \mathrm{x} 012000439 \mathrm{~B} 56$ FF51847E41B5728F9730D7B55F (Accessed Jan, 2020).

[26] "Image Data Compression," Report concerning Space Data System Standards, CCSDS 120.1-G-1-S, Green Book, Issue 1, Washington, D.C.: CCSDS, Jun. 2007.

\section{BIOGRAPHIES OF AUTHORS}

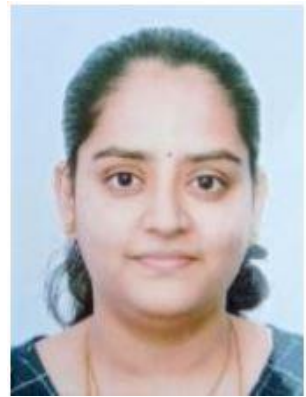

Gunasheela Keragodu Shivanna (D) 81 SC P received the B.E. degree in Electronics and Communication Engineering and the M. Tech. Degree in Signal processing from Visveswaraya technological University, India in the year 2012 and 2014, respectively. She is currently working towards the Ph.D. degree in hyperspectral image compression at Nitte Meenakshi institute of technology affiliated to VTU, India. Her research interests include compressed sensing, hyperspectral image processing, optimization, and computer vision. She can be contacted at email: gunasheela.ks.2012@gmail.com.

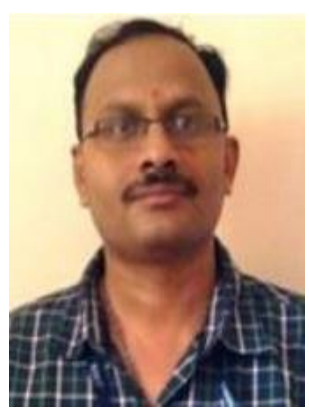

Haranahalli Shreenivasamurthy Prasantha (D) 8 SC $\mathrm{P}$ received bachelor's degree from Bangalore University, master's degree from V.T.U, Belgaum, and PhD from Anna University, Chennai, in Signal and Image Processing. He has 20+ years of teaching and research experience. His research interest includes Multimedia and Signal Processing. He has published more than 35 papers in international conferences and Journals. He is a reviewer for various reputed conferences and Journals. He is currently guiding four students for their research program under VTU. Currently, he is working as a professor in the department of Electronics and Communication Engineering, Nitte Meenakshi Institute of Technology (Affiliated to VTU Belgaum), Bangalore. He can be contacted at email: drhsprashanth@gmail.com. 